\title{
Fiber Bragg Grating Sensor Network Optimization
}

\author{
Guina WANG ${ }^{1,2 *}$, Jie ZENG ${ }^{1}$, Hao $\mathrm{MU}^{3}$, and Dakai LIANG ${ }^{1}$ \\ ${ }^{1}$ State Key Laboratory of Mechanics and Control of Mechanical Structures, Nanjing University of Aeronautics and \\ Astronautics, Nanjing, 210016, China \\ ${ }^{2}$ College of Mechanical and Electrical Engineering, Soochow University, Suzhou, 215021, China \\ ${ }^{3}$ Nanjing Jilong Optical Communication Co., Ltd, Nanjing, 210000, China \\ *Corresponding author: Guina WANGＥ-mail: ntyjxx@126.com
}

\begin{abstract}
This study introduces the optimization of the fiber Bragg grating (FBG) network and the load identification. Current researches on the optimal placement and reliability of the FBG network and the static load identification are generally analyzed. And then, the optimal placement of sensors and reliability of the FBG network are studied. Through the analysis of structural response characteristics, the general rules of sensors placement in structural static response parameters monitoring are proposed. The probability calculation is introduced, and the numerical analyses of the FBG sensor network reliability of several simple topologies are given.
\end{abstract}

Keywords: Fiber Bragg grating (FBG) sensor network, structural health monitoring (SHM), reliability, layout optimization, topology structure

Citation: Guina WANG, Jie ZENG, Hao MU, and Dakai LIANG, "Fiber Bragg Grating Sensor Network Optimization," Photonic Sensors, 2015, 5(2): 116-122.

\section{Introduction}

The fiber Bragg grating (FBG) sensor is a kind of mature sensor at present. We can construct the FBG sensor network based on FBG sensors through study on the characteristics. FBG sensors have several technical advantages in sensitivity, long-distance measurement, multiplexing of sensors, and immunity to electromagnetic noise compared with conventional electrical sensors [1].

Due to these advantages, the FBG has received considerable attraction to the structural health monitoring (SHM). The purpose of SHM is to monitor structural damages of an object [2], including: (1) whether there is any damage in the structure; (2) position determination and energy level assessment of the damage; (3) the extent of hazard to the structure. To determine whether there is any damage in the structure, we have to compare the monitoring data with the response of the structure health status. If the difference value is larger than a certain threshold on the premise of eliminate noise, the existence of damage can be determined. The key and difficult point for SHM is to identify the location of the damage, and after that, the damage level can be determined.

Two aspects should be considered in the design of FBG sensor network [3]: the using efficiency of sensors and the reliability of the FBG sensor network system. These two factors are opposite to some extent. On the premise of meeting the established requirements, a small number of sensors should be arranged in order to improve the system efficiency and reduce the cost. But in fact, we need

Received: 5 January 2015 / Revised version: 20 February 2015

(C) The Author(s) 2015. This article is published with open access at Springerlink.com

DOI: $10.1007 / \mathrm{s} 13320-015-0195-6$

Article type: Regular 
to improve the reliability of the sensor system through a variety of methods because of sensor failure or the optical fiber fracture. Therefore, the two factors should be considered in general during the study of FBG sensor network: forecast possible failures of the sensor network at a set time and then optimally design the FBG sensor network layout [4].

\section{FBG sensor network layout optimization}

\subsection{Sensor placement of one-dimensional beam structure}

The one-dimensional beam structure is mainly composed of the trusses structure and beam structure. Because the form of trusses under load is simple than that of the beam structure, we analyze the strain characteristics of the beam structure after loading by using the finite element analysis $[5,6]$ and research the sensor placement of the beam structural health monitoring according to the experiment data.

We arrange the location of the sensors according to the monitoring characteristics of physical quantities. And then the simulation analysis for the beam structure will be done to show structural response parameters.

A simply supported beam model is established by using the finite element analysis software ANSYS, where the beam material is aluminum alloy, the elasticity modulus $E=71 \mathrm{GPa}$, Poisson's ratio $v=0.31$, and the length, width, height are $500 \mathrm{~mm}, 20$ $\mathrm{mm}, 6 \mathrm{~mm}$, respectively. Twenty units are set along the beam length direction, and the length of each unit is $25 \mathrm{~mm}$. Each time one node is loaded with $100 \mathrm{~N}$ along $Y$ direction to obtain the response parameters of the beam structure. The displacement and strain after loading are shown in Fig. 1.

It can be concluded that in the beam structure, the location of the largest displacement is near the center, and the biggest strain is near the constraints. Therefore, for different forms of loading, the different paste positions of sensors would be in. As for the two-end clamped beam structure, the displacement is obvious in the middle of the area where the key point is, and the displacement is small near the constraint where the sensor response is not sensitive. When the research focuses on the structure's static response parameters, the location of the strain in the structure is on the loading place, but the negative strain is on the constraint place of the beam structure. Therefore, the sensors should be placed near the constraint for the analysis of the structure's static parameters.

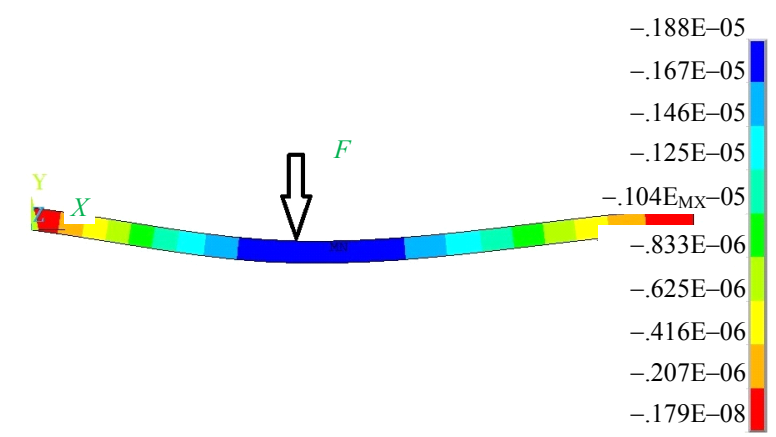

(a)

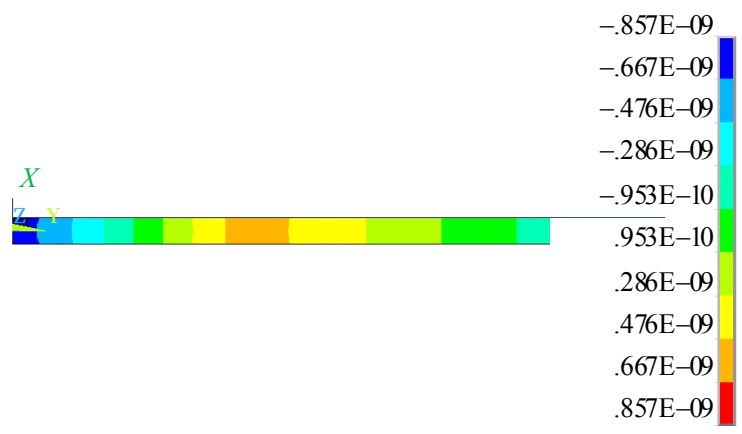

(b)

Fig. 1 Response of beam structure under static load: (a) displacement figure and (b) strain figure.

The loading points are chosen from $y=50 \mathrm{~mm}$ to $450 \mathrm{~mm}$ with steps of $50 \mathrm{~mm}$. The monitoring points are selected, $y=25 \mathrm{~mm}, 150 \mathrm{~mm}, 250 \mathrm{~mm}$, $350 \mathrm{~mm}$, and $475 \mathrm{~mm}$, respectively. The strain values of monitoring points after normalization processing are shown in Table 1.

The evaluation method commonly used for the optimal layout of the sensor is to determine the position according to the minimum correlation coefficient [7] and to arrange a redundant sensor according to the maximum comprehensive correlation coefficient. From the mathematical point 
of view, this method can get an optimal solution of math. The correlation coefficient is used to indicate the similarity between two vectors, which is expressed as

$$
r=\frac{n \sum x y-\sum x \sum y}{\sqrt{n \sum x^{2}-\left(\sum x\right)^{2}} \cdot \sqrt{n \sum y^{2}-\left(\sum y\right)^{2}}}
$$

where $r$ is the correlation coefficient, $x$ and $y$ are two variables, and $n$ is the number of each variable.

Table 1 Strain values of monitoring points after normalization processing.

\begin{tabular}{cccccc}
$\begin{array}{c}\text { Monitoring } \\
\text { points(mm) } \\
\text { Loading } \\
\text { location(mm) }\end{array}$ & $\begin{array}{c}\text { No. 1 } \\
y=25\end{array}$ & $\begin{array}{c}\text { No. 2 } \\
y=150\end{array}$ & $\begin{array}{c}\text { No. 3 } \\
y=250\end{array}$ & $\begin{array}{c}\text { No. 4 } \\
y=350\end{array}$ & $\begin{array}{c}\text { No. 5 } \\
y=475\end{array}$ \\
\hline$y=50$ & 0.28 & -0.10 & -0.05 & 0.01 & 0.07 \\
$y=100$ & 0.73 & -0.40 & -0.19 & 0.01 & 0.24 \\
$y=150$ & 0.96 & -0.81 & -0.44 & -0.02 & 0.47 \\
$y=200$ & 1.00 & -0.49 & -0.79 & -0.09 & 0.70 \\
$y=250$ & 0.90 & -0.24 & -1.17 & -0.24 & 0.90 \\
$y=300$ & 0.70 & -0.09 & -0.79 & -0.49 & 1.00 \\
$y=350$ & 0.47 & -0.02 & -0.44 & -0.81 & 0.96 \\
$y=400$ & 0.24 & 0.01 & -0.19 & -0.40 & 0.73 \\
$y=450$ & 0.07 & 0.01 & -0.05 & -0.10 & 0.28 \\
\hline
\end{tabular}

After calculation according to Table 1, the correlation coefficients of monitoring points are shown in Table 2.

Table 2 Strain correlation coefficient of monitoring points.

\begin{tabular}{cccccc}
\hline $\begin{array}{c}\text { Monitoring } \\
\text { points (mm) } \\
\text { location }(\mathrm{mm})\end{array}$ & $\begin{array}{c}\text { No. 1 } \\
y=25\end{array}$ & $\begin{array}{c}\text { No. } 2 \\
y=150\end{array}$ & $\begin{array}{c}\text { No. } 3 \\
y=250\end{array}$ & $\begin{array}{c}\text { No. } 4 \\
y=350\end{array}$ & $\begin{array}{c}\text { No. 5 } \\
y=475\end{array}$ \\
\hline$y=25$ & 1 & -0.7916 & -0.7363 & 0.1675 & 0.3380 \\
$y=150$ & -0.7916 & 1 & 0.2430 & -0.5463 & 0.1675 \\
$y=250$ & -0.7363 & 0.2430 & 1 & 0.2430 & -0.7363 \\
$y=350$ & 0.1675 & -0.5463 & 0.2430 & 1 & -0.7916 \\
$y=475$ & 0.3380 & 0.1675 & -0.7363 & -0.7916 & 1 \\
\hline
\end{tabular}

When arranging two sensors in the beam structure, the optimal placement should be selected by the maximum difference of the relevant coefficient of the two loaded points. We can see from Table 2 that the placement of monitoring points should be No. 1 and No. 2 or No. 3 and No. 5 .

Directly analyzing the data in Table 2 , the strain of monitoring No. 1 and No. 5 is always bigger than that of monitoring No. 2, No. 3, and No. 4 in the process of each loading, and the value of measured strain maintains plus or minus in the process of moving loading points. So the combination of No. 1 and No. 5 is an optimal solution for beam structural strain monitoring.

\subsection{Sensor placement of four edges clamped two-dimensional plate structure}

Taken a four-edge clamped plate for an example [8], according to the finite element simulation analysis, the total strain nephogram of the plate structure is shown in Fig. 2.

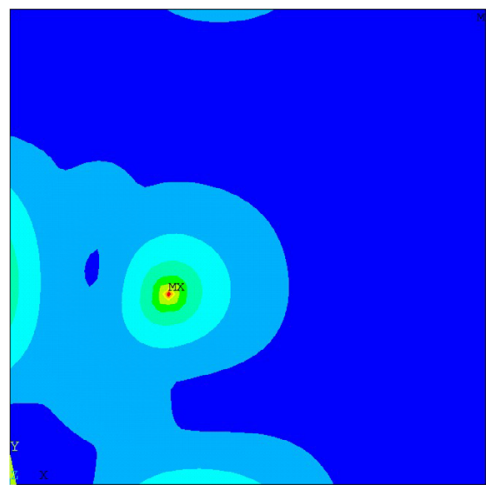

Fig. 2 Total strain nephogram of the plate structure.

It can be seen from Fig. 2 that the same as one-dimensional structural, negative strain exists on the side of constraint place under static loading, and the location of positive strain is near the loading place. Therefore, it can be concluded that the same as one-dimensional structure, the place near the constraint can be used as an optional strain monitoring point.

\section{Reliability of optical FBG sensor network}

When the optical FBG placement is determined, we should confirm the network mode and design the monitoring system according to the principle of maximum reliability. At present, for FBG sensor networks, the optical FBG is suitable for single-channel multipoint measurement by means of wavelength division multiplex (WDM). Considering the sensor network topology form, the optical FBG is especially suitable for the monitoring network in the form of series and parallel composition. The structure of sensor network topology optimization can improve the monitoring system on node damage tolerance and the system of self-healing capability [9]. 


\subsection{Reliability theory of the measurement system}

The reliability $[10,11]$ of the measurement system is an effective monitoring ability to the object within some certain time. The measurement system is mainly composed of measurement units, mediation units, information transmission, and processing units. The failure of any function of the units will lead to part or all functional failure of the measurement system. So the research on the reliability of measurement system is not only to improve the reliability of a single unit, but also to optimize the design on the system and reduce the impact by the failure unit.

\subsection{Optical FBG sensor network reliability research based on the probability analysis}

The damage probability of each node is determined according to the arrangement characteristics of fiber grating sensor network [12]. For the FBG, the grating area may be failure due to the high temperature, but there will not be fracture failure after encapsulation. For the transmission optical fiber, the form of failure generally is fracture failure that can break off the light path. Therefore, for optical FBG monitoring system reliability, the failure reasons are mainly grating failure and the fracture failure.

By symbolizing optical FBG failure probability with $P_{1}$ and the optical fiber fracture probability with $P_{2}$, the corresponding reliable probability is $\left(1-P_{1}\right)$ and $\left(1-P_{2}\right)$, respectively [13]. Through symbolizing the failure probability of the whole system with $P$, the corresponding failure probability is determined by the optical FBG network topology model.

Fig. 3 shows the simplest topological structure of fiber grating network [14]. It is the most common optical FBG in series in actual projects and experiments.

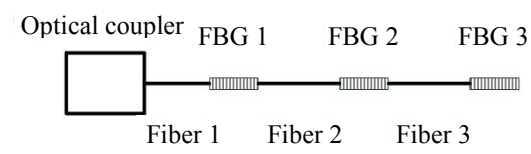

Fig. 3 Optical FBG placement in series.
The reliability of the measurement system shown in Fig. 3 can be expressed as

$$
P_{0}=\left(1-P_{1}\right)^{3}\left(1-P_{2}\right)^{3}
$$

where $P_{0}$ is the lossless probability that can keep sensing grating sensor network in a good condition.

But it cannot completely describe the reliability of the FBG sensing system. So it is necessary for the FBG sensor network to define the system integrity, which is expressed in $T$ that reflects the proportion of connected FBG after damage in the fiber grating sensor network system. As shown in Fig. 3, if the FBG monitoring system breaks off in point Fiber 2, and there is only one connected FBG, the integrity $T_{f 1}$ of the system is $33 \%$.

If there is one breakpoint, the expected integrity of the system is [15]

$$
E(T)=P_{1} T_{F 1}+P_{1} T_{F 2}+P_{1} T_{F 3}+P_{2} T_{f 1}+P_{2} T_{f 2}+P_{2} T_{f 3}
$$

where $T_{f}$ is the system integrity after fracture failure, and $T_{F}$ is the system integrity after failure for corresponding transmission optical fiber. When the series number of grating is n, Eq. (3) can be expressed as

$$
E(T)=P_{1} \sum T_{F i}+P_{2} \sum T_{f i}
$$

When the system breakpoints increase, the expected integrity can be obtained by using Eq. (4) repeatedly.

At normal temperatures, SHM is mainly aimed at the strain characteristics. For the FGB monitoring system, the possible damage area mostly happens in the part of transmission because there is no degeneration in the grating area at normal temperatures. Under this condition, the expected integrity of the system is

$$
E(T)=P_{2} \sum T_{f i}
$$

As we know, the FBG sensor is a two-way transmission sensor. If connecting a tail fiber to the demodulation instrument from the end of series sensors, as shown in Fig. 4, once there is a breakpoint occurred in sensor network at this time, 
the FBG signal can still be read. Therefore, a breakpoint will not destroy the integrity of the network in the ring system. When two breakpoints occur in the sensor network, the expected integrity of the system is: $E(T)=0.5 P_{2} \cdot P_{2}$.

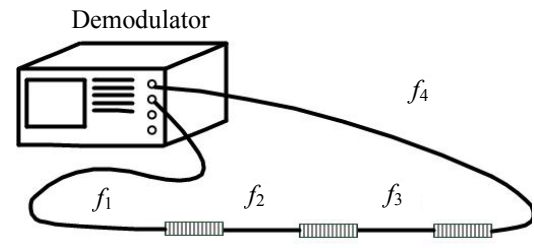

Fig. 4 Ring series layout of FBG sensors.

In fact, if the probability of one breakpoint occurred in the monitoring system is 0.05 , we can conclude from the comparison between above two methods that the complete failure probability is 0.025 and 0.00125 in the series system and ring network system, respectively. The reliability of the ring system increases 20 times.

Several series branches can be connected to a fiber Bragg grating demodulation instrument in the form of parallel using optical coupler. As shown in Fig. 5.

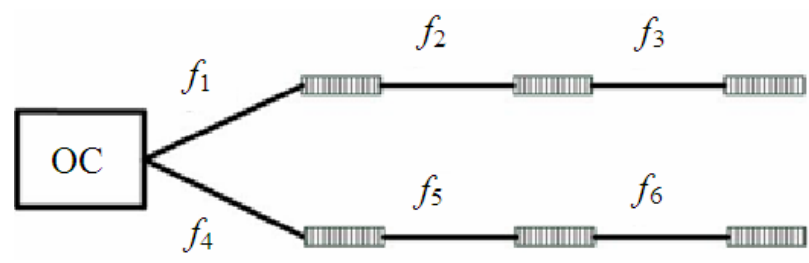

Fig. 5 Parallel layout of FBG sensors.

According to the characteristics of structure topology, system integrity can be calculated when breakpoints occur on each part of the optical fiber. According to Eq. (3), if there is one breakpoint, the expected integrity of the system is $E(T)=0.25 P_{2}$. Compared with series layout, the system integrity of parallel layout is twice, so it is an optimal topology structure.

For the composite FBG network, the performance of a single FBG sensor can greatly be improved through an additional connected light path. The reliability of the sensor will increase highly if designing a redundancy light path for a single sensor, such as the layout shown in Fig. 6.

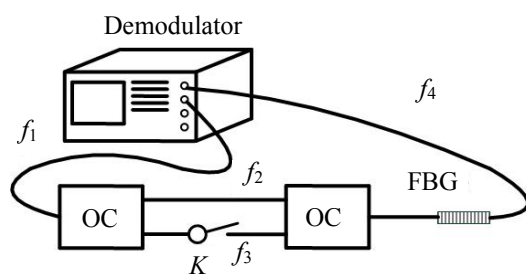

Fig. 6 Redundancy light path layout.

In Fig. 6, there are two optical fibers in parallel between two optical couplers. According to Eq. (3), when the optical fiber routing is composed of $f_{1}, f_{2}$, and $f_{4}$, the integrity expectation is $E(T)=1$. When the FBG is connected with a ring routing, the single breakpoint occurring on the transmission optical fiber will not affect the integrity of monitoring system. Considering two breakpoints existing in the system, there are six kinds of possible combinations, and only $f_{1}, f_{4}$, and $f_{2}, f_{4}$ have the possibility of destroying the system. Under this condition, the expected integrity of the system is $E(T)=0.33 P_{2} \cdot P_{2}$.

Supposing that a certain optical fiber is under severe working conditions or it plays important roles in the monitoring system, it can be designed as shown in Fig. 6 to increase the reliability, and now the expected integrity is $E(T)=0.17 P_{2} \cdot P_{2}$.

\subsection{Optical fiber sensor network reliability optimization}

At present, the method of improving the reliability through the fiber grating sensor network topology optimization is mainly aimed at the optimization on FBG sensor node failure [16] and the improvement of the reliability through designing redundant FBGs and connecting the light routing. Actually, the transmission optical fiber and FBG grating area are made of quartz with the coating layer, and the FBG grating area normally has a special encapsulation structure that can improve the security more effective than the transmission optical fiber. So we present a network-ring topology fiber 
grating topology structure, as shown in Fig. 7.

In Fig. 7, dashed lines mean arranging redundant light routings in the ring main net node.

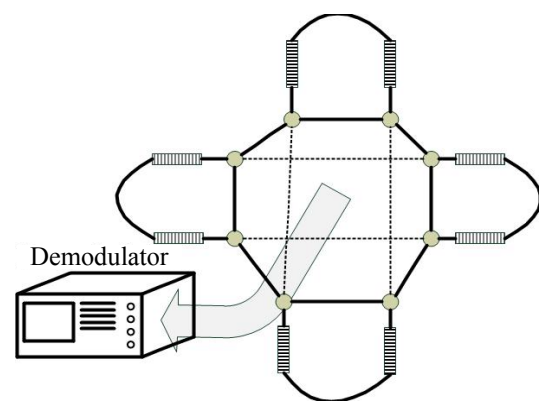

Fig. 7 Network - ring topology structure layout.

The subnet of the FBG sensor connects to the main ring through the couplers in the Fig. 6. For one FBG, it will completely fail only when failure or fracture occurs so that it is unable to connect to the couplers. For the ring network, if there are two breakpoints in the main net, the failure FBG expectation is half of the total number of FBG. Therefore, it is more harmful that two breakpoints occur in the ring main net than the case in subnet of the FBG. During the process of designing the sensor network topology optimization of FBG, we should focus on improving the reliability of the main net. Reliability of the optical fiber sensor network should be improved by increasing the reliability of the main net.

\section{Conclusions}

We introduce the advantages of FBG and study the FBG sensor layout through analyzing the static response in the beam structure and four-edge clamped plate structure. We can conclude that when the monitoring object is strain, the sensor should be arranged at the constraint point, but when the monitoring object is displacement, the sensor should be arranged in the area where the structure deformation is relatively larger. When the position of the sensor is settled, the connection form should be arranged, and the reliability should be analyzed. We go on studying on the reliability of the FBG in different network forms by using the method of probability and taking the influence on sensor network when breakpoints occur as the reliability assessment index of a sensor network topology structure.

\section{Acknowledgement}

This work was supported by the Chinese National Natural Science Foundation (Grant No. 51275239, 11402112), the Aerospace CAST Innovation Foundation, the Cooperative Innovation Foundation of Jiangsu Province (Grant No. 2014003-01), the Postdoctoral Science Foundation (Grant No. 20090461116), the Aviation Science Foundation (Grant No. 20125652055), the Doctor Research Foundation (Grant No. 20123218110003). We also thank the State Key Laboratory of Mechanics and Control of Mechanical Structures of Nanjing University of Aeronautics and Astronautics.

Open Access This article is distributed under the terms of the Creative Commons Attribution License which permits any use, distribution, and reproduction in any medium, provided the original author(s) and source are credited.

\section{References}

[1] J. Lee, S. Lee, and D. Yoon, "Simultaneous multipoint acoustic emission sensing using fibre acoustic wave grating sensors with identical spectrum," Journal of Optics A: Pure and Applied Optics, 2008, 10(8): 085307-1-085307-3.

[2] S. N. Vecherin, D. K. Wilson, and C. L. Pettit, "Optimal sensor placement with terrain-based constraints and signal propagation effects," in Proc. SPIE, vol. 7333, pp. 73330S-1-73330S-12, 2009.

[3] S. Saad and L. Hassine. "Hydrogen detection with FBG sensor technology for disaster prevention," Photonic Sensors, 2013, 3(3): 214-223.

[4] C. K. Y. Leung, K. T. Wan, D. Inaudi, X. Bao, W. Habel, Z. Zhou, et al., "Michio imai review: optical fiber sensors for civil engineering applications," Materials and Structures, 2013, 48(4): pp. 871-906.

[5] G. Tsamasphyros, N. Furnarkis, G. Kanderakis, and Z. Marioli-Riga, "Optimization of embedded optical sensor location in composite repairs," Applied Composite Materials, 2003, 10(3): 129-140.

[6] T. Hisada, "Recent progress in nonlinear FEM-based 
sensitivity analysis," JSME International Journal Series A, 1995, 38(3): 301.

[7] Z. Du, "The relationship between prediction accuracy and correlation coefficient," Solar Phys, 2011, 270(1): 407-416.

[8] Y. M. Grigorenko and A. Y. Grigorenko, "Static and dynamic problems for anisotropic inhomogeneous shells with variable parameters and their numerical solution," International Applied Mechanics, 2013, 49(2): 123-193.

[9] A. Panopoulou, S. Fransen, V. Gomez-Molinero, and V. Kostopoulos, "Experimental modal analysis and dynamic strain fiber Bragg gratings for structural health monitoring of composite antenna sub-reflector," CEAS Space Journal, 2013, 5(1-2): 57-73.

[10] V. N. Stroitelev, P. G. Shabanov, and T. V. Shaposhnikova, "Effects of error variation in means of measurement on reliability parameters in engineering-system monitoring," Measurement Techniques, 1994, 37(1): 18-21.

[11] F. Berghmans, S. Eve, and M. Held, "An introduction to reliability of optical components and fiber optic sensors," in Optical Waveguide Sensing and Imaging NATO Science for Peace and Security Series 2008, pp. 73-100, 2008.

[12] A. Othonos, "Bragg gratings in optical fibers: fundamentals and applications," in Optical Fiber Sensor Technology 2000, pp. 79-187, 2000.

[13] S. Bhalla, Y. W. Yang, and C. K. Soh. "Monitoring of rocks and underground structures using PZT and FBG sensors," in Smart Materials in Structural Health Monitoring Control and Biomechanics Advanced Topics in Science and Technology in China 2012, pp. 481-499, 2012.

[14] Y. Gong, C. Gan, C. Wu, and R. Wang, "Novel cobweb-topology WDM access network architecture featuring ultra-high reliability and easy scalability," Optical and Quantum Electronics, 2014, 46(8): 999-1019.

[15] E. Rizk, H. Marzouk, A. Hussein, and X. Gu, "Structural health monitoring of slab-column connections using FBG sensors," Journal of Civil Structural Health Monitoring, 2012, 2(1): 17-27.

[16] H. Murayama, D. Wada, and H. Igawa, "Structural health monitoring by using fiber-optic distributed strain sensors with high spatial resolution," Photonic Sensors, 2013, 3(4): 355-376. 